\title{
Multiplicative Dynamics Underlie the Emergence of the Log- Normal Distribution of Spine Sizes in the Neocortex In Vivo
}

\author{
Yonatan Loewenstein, ${ }^{1}$ Annerose Kuras,${ }^{2 \dagger}$ and Simon Rumpel ${ }^{2}$ \\ ${ }^{1}$ Department of Neurobiology, Edmond and Lily Safra Center for Brain Sciences, Interdisciplinary Center for Neural Computation and Center for the Study \\ of Rationality, Hebrew University, Jerusalem 91904, Israel, and 2IMP—Research Institute of Molecular Pathology, Vienna 1030, Austria
}

What fundamental properties of synaptic connectivity in the neocortex stem from the ongoing dynamics of synaptic changes? In this study, we seek to find the rules shaping the stationary distribution of synaptic efficacies in the cortex. To address this question, we combined chronic imaging of hundreds of spines in the auditory cortex of mice in vivo over weeks with modeling techniques to quantitatively study the dynamics of spines, the morphological correlates of excitatory synapses in the neocortex. We found that the stationary distribution of spine sizes of individual neurons can be exceptionally well described by a log-normal function. We furthermore show that spines exhibit substantial volatility in their sizes at timescales that range from days to months. Interestingly, the magnitude of changes in spine sizes is proportional to the size of the spine. Such multiplicative dynamics are in contrast with conventional models of synaptic plasticity, learning, and memory, which typically assume additive dynamics. Moreover, we show that the ongoing dynamics of spine sizes can be captured by a simple phenomenological model that operates at two timescales of days and months. This model converges to a log-normal distribution, bridging the gap between synaptic dynamics and the stationary distribution of synaptic efficacies.

\section{Introduction}

It is generally believed that the structure and plasticity of synaptic connectivity in the neocortex underlies much of our ability to process and store information. The prevailing view is that changes in the efficacies of synapses in the cortex are determined by the presynaptic and postsynaptic activities. Therefore, these efficacies reflect the mental history of the animal. Intriguingly, previous studies have shown that the distribution of connection strengths in the cortex is skewed (Feldmeyer et al., 2002; Arellano et al., 2007). Some studies approximated this distribution using a log-normal function (Song et al., 2005; Sarid et al., 2007). However, how this skewed distribution of efficacies emerges is an open question.

In recent years, chronic spine imaging has opened the door to studying these dynamics. Most of the excitatory synapses onto pyramidal cortical neurons reside on dendritic spines. The presence of a spine is therefore indicative of an excitatory synapse, and previous studies used this fact to study the turnover of synapses in the living brain, primarily considering spines as binary entities (Grutzendler et al., 2002; Trachtenberg et al., 2002; Oray et al., 2004; Holtmaat et al.,

Received Nov. 23, 2010; revised March 22, 2011; accepted May 8, 2011.

Author contributions:S.R. designed research;S.R. performed research;Y.L., A.K., and S.R. analyzed data; Y.L. and S.R. wrote the paper.

This work was supported by The Israel Science Foundation (Grant No. 868/08 to Y.L.), a grant funded by the German-Israeli Foundation for Scientific Research and Development (to Y.L.), the Gatsby Charitable Foundation (to Y.L.), and the Boehringer Ingelheim (A.K., S.R.). We thank Anthony Holtmaat and Karel Svoboda for advice, Tony Zador and Robert Malinow for support and encouragement, Ariel More for technical help, and Eran Marom, Sen Song, and Brice Bathellier for helpful comments on the manuscript.

${ }^{\dagger}$ Deceased May 11, 2008

The authors declares no competing financial interests.

Correspondence should be addressed to Simon Rumpel, IMP—Research Institute of Molecular Pathology, Dr. Bohr-Gasse 7, Vienna 1030, Austria, E-mail: rumpel@imp.ac.at; or Yonatan Loewenstein, Hebrew University, Jerusalem 91904, Israel, E-mail: yonatan@huji.ac.il.

DOI:10.1523/JNEUROSCI.6130-10.2011

Copyright $\odot 2011$ the authors $\quad 0270-6474 / 11 / 319481-08 \$ 15.00 / 0$
2005; Zuo et al., 2005; Majewska et al., 2006; Bhatt et al., 2009; Holtmaat and Svoboda, 2009; Xu et al., 2009; Yang et al., 2009). These studies have shown that while some spines appear and disappear throughout adult life, a considerable fraction of the spines persists for extended periods of time (Bhatt et al., 2009; Holtmaat and Svoboda, 2009). These spines have been referred to as "stable" and are the likely locus of the synapses that underlie long-term memories.

There is substantial evidence that the size of a spine provides information about the efficacy of the synapse that resides on it: the volume of a spine is proportional to (1) the number of vesicles in the presynaptic terminal (Harris and Stevens, 1989); (2) the area of the postsynaptic density (Harris and Stevens, 1989; Knott et al., 2006; Arellano et al., 2007; Katz et al., 2009); (3) the number of postsynaptic AMPA receptors (Katz et al., 2009); and (4) the amplitude of the EPSC measured in the postsynaptic neuron following uncaging of glutamate near the spine (Matsuzaki et al., 2001). Furthermore, long-term potentiation of synapses is associated with an increase in the volume of spines (Matsuzaki et al., 2004; Kopec et al., 2006). Previous studies have shown that spine sizes are correlated with spine age (Holtmaat et al., 2006) and that average spine sizes can change following sensory deprivation (Hofer et al., 2009). However, a quantitative description of the dynamics of spine sizes has been lacking.

We analyzed the stationary distribution and temporal dynamics of the sizes of hundreds of "stable" spines. We show that the size distribution of spines of individual neurons is log-normally distributed and that there is only little heterogeneity in this distribution between neurons. Although this distribution is stable over time, individual spines show substantial changes in their size. Interestingly, the magnitude of the change in size of a spine is, on average, proportional to its size. Such multiplicative dynamics explain the emergence of a log-normal distribution of spine sizes. Finally, we show that the temporal changes in spine size can be well approximated in a model characterized by two timescales $(\sim 3 \mathrm{~d}$ and $\sim 200 \mathrm{~d})$. 


\section{Materials and Methods}

In vivo two-photon spine imaging. The procedures for implantation of a glass window in the cranium and subsequent in vivo two-photon imaging have been described in detail previously (Holtmaat et al., 2009). Briefly, six male adult ( $\sim 6$ months) in-house bred mice of the GFP-M transgenic line selected for extremely sparse GFP expression in the cortex (Feng et al., 2000) were deeply anesthetized with a single dose of ketamine/medetomidine. After removal of an $\sim 1 \mathrm{~cm}^{2}$ patch of skin over the parietal bones and right lateral part of the head, a small part of the musculus temporalis was removed to expose the temporal bone. Using a dentist's drill, the bones were smoothened, and part of the zygomatic process was removed and covered with a thin layer of Vetbond glue (3M). Next, a thin layer of dental cement (Lang Dental) was applied, except for the area over the temporal bone. The temporal bone was removed with a dental drill to expose a $\sim 2 \mathrm{~mm} \times 3 \mathrm{~mm}$ part of the brain containing the auditory cortex. The craniotomy was subsequently sealed with a drop of liquid agarose, a small round cover glass, and additional dental cement. In addition, a small titanium head post was implanted for fixation of the head during imaging. After recovery from the surgery and anesthesia, the mice were brought back to their home cage and regular imaging sessions were started 2 weeks after the surgery.

For imaging, mice were anesthetized lightly with a single dose of ketamine/medetomidine and fixated under an Olympus FV300 microscope custom modified for two-photon imaging. We used a Chameleon Ti:Sapphire laser (Coherent) tuned to $910 \mathrm{~nm}$ wavelength for excitation of the GFP. Image stacks of dendritic segments were acquired using FluoView image acquisition software (Olympus) with typically 20-30 frames $(512 \times 512$ pixels, 1 pixel $\approx 0.1 \mu \mathrm{m} \times 0.1 \mu \mathrm{m}, \sim 1.7 \mathrm{~s} /$ frame) at an interval of $0.5 \mu \mathrm{m}$ along the $z$-axis. Imaging stacks along a dendrite were acquired in an overlapping manner, and as a result, some of the spines were imaged twice. All the "stable" spines that were imaged twice were used to estimate the within-session variability (see Fig. 5). Imaging was performed at an interval of $4 \mathrm{~d}$ for six time points. A single imaging session typically lasted about $1 \mathrm{~h}$. Between imaging sessions, mice were housed in small groups in standard cages. A subset of the mice underwent a classical conditioning protocol between sessions 3 and 4; however, no gross differences were observed between the two datasets and therefore for the purpose of this study data from both groups were pooled together. All experiments were performed at Cold Spring Harbor Laboratory in strict compliance with the animal use and care guidelines of Cold Spring Harbor Laboratory.

Primary image analysis of spines involved two steps. First, "best projections" of all dendrites for all time points were constructed, allowing for the identification of spines at a given time point and the indexing of identical spines along time (Holtmaat et al., 2005). We did not attempt to distinguish between spines and filopodia and classified all protrusions from the dendrite as spines for the purpose of our study. Second, custom-written Matlab (MathWorks) software was used to extract image features of identified spines. We only considered spines that extended from the dendrite in parallel to the imaged plane. The analysis was performed in two dimensions at the image frame that gave highest integrated fluorescence values for a given spine, i.e., the frame where the spine was in focus. Each frame was filtered with a $3 \times 3$ pixel median filter, and then the background was subtracted. For estimation of spine sizes, we integrated the filtered fluorescence values within a region of interest containing the spine. This integrated fluorescence value was normalized by the mean intensity value of the brightest $10 \%$ of the pixels that was found on the adjacent dendrite, which typically is a very stable feature of the image. This normalization compensates for possible variances in the general brightness of the image and possible differences in expression levels of GFP between neurons. A previous study performing electron microscopy on serial sections of previously in vivo imaged fluorescent dendrites demonstrated that such integrated fluorescence is a high-fidelity proxy for the size of a spine (Holtmaat et al., 2005).

Fitting the parameters of the model of the dynamics of spine sizes. We describe the dynamics of the logarithm of the size of a spine as a sum of two independent Ornstein-Uhlenbeck processes:

$$
\begin{gathered}
\tau_{i} \dot{X}_{i}^{k}=-X_{i}^{k}+\xi_{i}^{k} \quad i=1,2 \\
\log _{10}\left(S^{k}\right)=X_{1}^{k}+X_{2}^{k}+\mu,
\end{gathered}
$$

where $S^{k}$ is the size of the spine, $X_{1}^{k}$ and $X_{2}^{k}$ are the two dynamic variables whose dynamics are described by an Ornstein-Uhlenbeck process, $\xi_{i}^{k}$ is a white noise term such that $\left\langle\xi_{i}^{k}\right\rangle=0$ and $\left\langle\xi_{i}^{k}(t) \xi_{j}^{k^{\prime}}\left(t^{\prime}\right)\right\rangle=2 \tau_{i} \sigma_{i}^{2} \delta_{i, j} \delta_{k, k^{\prime}} \delta(t-$ $\left.t^{\prime}\right)$, where $\delta_{i, j}=\left\{\begin{array}{ll}1, & \text { if } i=j \\ 0, & \text { if } i \neq j\end{array}\right.$ is the Kronecker delta and $\delta\left(t-t^{\prime}\right)$ is Dirac delta "function". The constant $\mu=1.74$ is the average of the logarithm of all spine sizes; the values of $\tau_{i}$ correspond to the timescales of the two processes and $\sigma_{i}^{2}$ are the stationary variances of $X_{i}$.

We further assume that the logarithm of the observed size of spine $k$, $O^{k}$, at time $t$ is a sum of these two Ornstein-Uhlenbeck processes and a measurement noise term, $n^{k}$, such that $\log _{10}\left(O^{k}(t)\right)=\log \left(S^{k}(t)\right)+n^{k}(t)$, where $\left\langle n^{k}(t)\right\rangle=0$ and $\left\langle n^{k}(t) n^{k^{\prime}}\left(t^{\prime}\right)\right\rangle=\sigma_{n}^{2} \delta_{k, k^{\prime}} \delta_{t, t^{\prime}}$ (we assume that the temporal correlations in the measurement noise are much shorter than the $4 \mathrm{~d}$ interval between measurements). In Results, we use Figure $5 B$ to estimate the measurement noise, and our analysis yields that $\sigma_{n}^{2}=$ $2.74 \cdot 10^{-3}$. Thus, comparing the total variance in the logarithm of the spine sizes over all "stable" spines and over all days, we conclude that the contribution of the measurement noise to the observed variance in the distribution of spine sizes is $<3 \%$ (see Results).

To find the parameters characterizing the two Ornstein-Uhlenbeck processes, $\tau_{1}, \tau_{2}, \sigma_{1}$, and $\sigma_{2}$, we considered the covariance function $C(\Delta t)=\left\langle\left(\log _{10}(O(t+\Delta t))-\left\langle\log _{10}(O(t+\Delta t))\right\rangle\right) \cdot\left(\log _{10}(O(t))-\right.\right.$ $\left.\left.\left\langle\log _{10}(O(t))\right\rangle\right)\right\rangle$, where the average $\langle\cdots\rangle$ is the average over time and over the empirical distribution of spines. It is easy to show that

$$
C(\Delta t)=\sigma_{n}^{2} \delta_{\Delta t, 0}+\sigma_{1}^{2} e^{-\frac{|\Delta t|}{\tau_{1}}}+\sigma_{2}^{2} e^{-\frac{|\Delta t|}{\tau_{2}}} .
$$

The parameters $\tau_{1}, \tau_{2}, \sigma_{1}$, and $\sigma_{2}$ were found by a least-square fit of $C(\Delta t)$ to the empirical covariance function (see Fig. $6 A$ ), yielding $\tau_{1}=212 \mathrm{~d}$; $\tau_{2}=2.87 \mathrm{~d} ; \sigma_{1}^{2}=0.0683$; and $\sigma_{2}^{2}=0.0292$.

Concerned about the possible consequences of underestimating the measurement noise, we theoretically studied how underestimating the measurement noise would affect the estimated parameters of the model. To do so, we fitted the parameters of the model to our data assuming that the measurement noise is double the value reported in the text $\left(\sigma_{n}^{2}=2 \times 2.74 \cdot 10^{-3}\right)$. We found that this would change the estimated values of $\tau_{1}, \tau_{2}, \sigma_{1}^{2}$, and $\sigma_{2}^{2}$ by only $-10 \%,+1 \%,+1 \%$, and $-11 \%$, respectively. Thus, we conclude that even an underestimation of the measurement noise by a factor of two would not have qualitatively affected the conclusions of the paper.

To compute the transition matrix (see Fig. 6C), we assumed that the Ornstein-Uhlenbeck processes reached their stationary distribution. A straightforward calculation yields that the probability of observing the size of a spine $O^{\prime}$ at time $t+\Delta t$ given that at time $t$ its size is $O$ is given by

$$
\operatorname{Pr}\left[\log _{10}\left(O^{\prime}\right) ; t+\Delta t \mid \log _{10}(O) ; t\right]=\frac{e^{-\frac{\left(\log _{10}\left(O^{\prime}\right)-m\right)^{2}}{2 \sigma_{\sigma o}^{2}}}}{\sqrt{2 \pi \sigma_{O^{\prime} O}^{2}}},
$$

where $\beta=\frac{\sigma_{1}^{2} e^{-\frac{\Delta t}{\tau_{1}}}+\sigma_{2}^{2} e^{-\frac{\Delta t}{\tau_{2}}}}{\sigma_{O}^{2}}, \sigma_{O^{\prime} O}^{2}=\sigma_{O}^{2}\left(1-\beta^{2}\right), \sigma_{O}^{2}=\sigma_{1}^{2}+\sigma_{2}^{2}+\sigma_{n}^{2}$, and $m=\beta \cdot \log _{10}(O)+(1-\beta) \cdot \mu$.

To construct the predicted dependence of absolute change on spine size (Fig. $3 C$, red line), we use Equation 3 to show that

$$
\operatorname{Pr}\left[O^{\prime} \mid O\right]=\frac{1}{O^{\prime}} \cdot \frac{e^{-\frac{\left(\log \left(O^{\prime}\right)-\left(\beta \log (O)+(1-\beta) \mu^{\prime}\right)^{2}\right.}{2\left(\sigma_{\sigma \sigma^{\prime}}^{e}\right.}}}{\sqrt{2 \pi\left(\sigma_{O^{\prime} O}^{e}\right)^{2}}},
$$

where $\mu^{e}=\mu / \log _{10}(e)$ and $\sigma_{O^{\prime} O}^{e}=\sigma_{O^{\prime} O} / \log _{10}(e)$. Averaging over $O^{\prime}$ yields the following:

$$
\begin{aligned}
& \mathrm{E}\left[\left(\left|O^{\prime}-O\right|\right) \mid O\right]=O \operatorname{erf}\left(\frac{(1-\beta)\left(\log (O)-\mu^{e}\right)}{\sqrt{2} \sigma_{O^{\prime} O}^{e}}\right) \\
& -e^{\beta \log (O)+(1-\beta) \mu^{e}+\frac{\left(\sigma_{\left.\sigma^{e}\right)^{2}}^{2}\right.}{2}} \operatorname{erf}\left(\frac{(1-\beta)\left(\log (O)-\mu^{e}\right)-\left(\sigma_{O^{\prime} O}^{e}\right)^{2}}{\sqrt{2} \sigma_{O^{\prime} O}^{e}}\right) .
\end{aligned}
$$



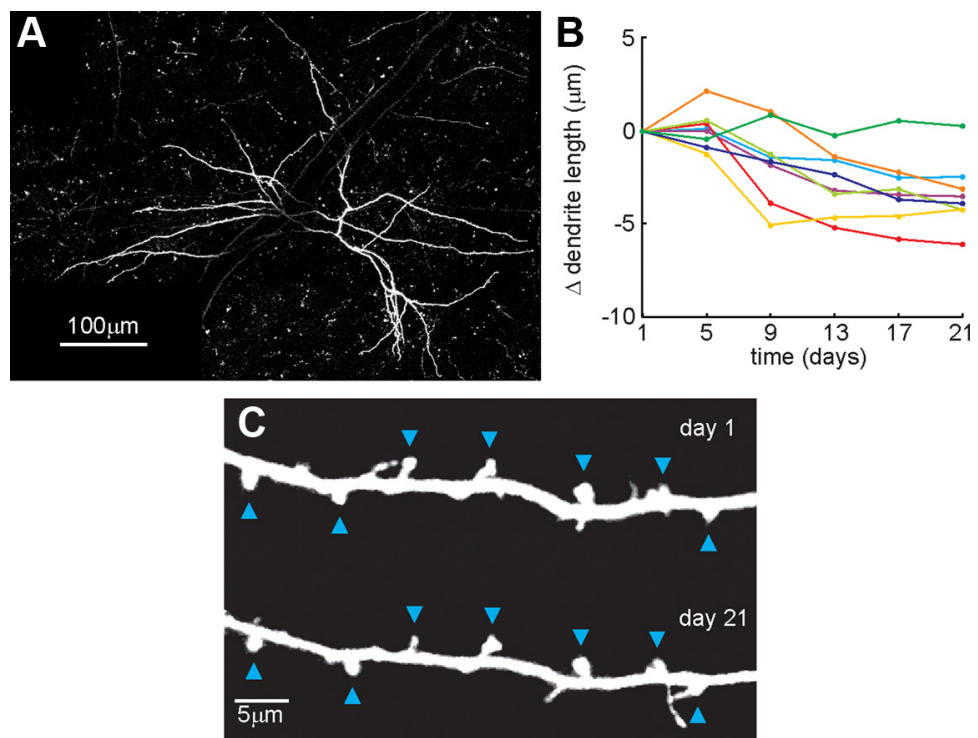

Figure 1. In vivo imaging of dendrites and spines. $\boldsymbol{A}$, Maximum intensity projection of image stacks taken from an apical dendrite of a GFP-expressing neuron in the auditory cortex. $\boldsymbol{B}$, Mean change of dendrite length per neuron ( $n=3-12$ per neuron, colored lines). C, Top, A "best projection" image (see Material and Methods) of the tip of a dendrite on the first imaging day. Bottom, Same dendrite on the last imaging day. Spines present at both time points are marked with blue arrowheads. Colors for individual neurons are the same as in Figure 3.
A
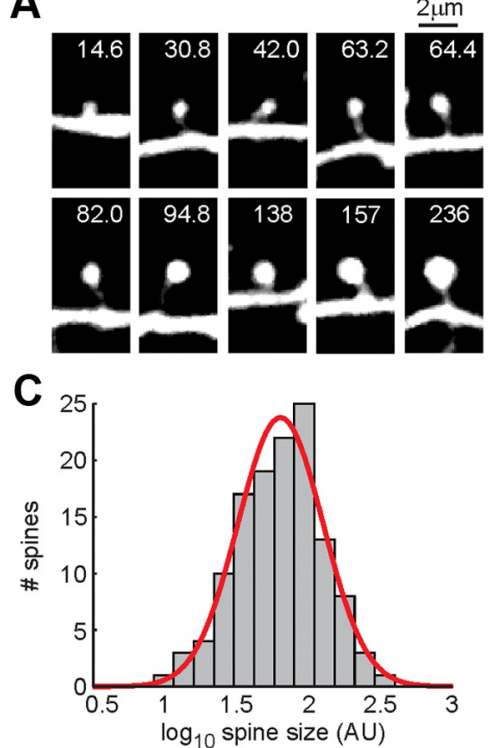
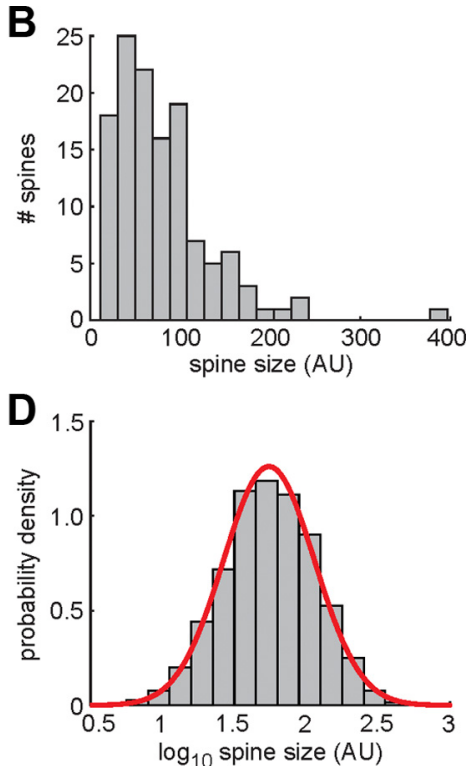

Figure 2. Log-normal distribution of spine sizes. $\boldsymbol{A}$, Examples of spines imaged along the dendrites of one neuron in one imaging session. The numbers indicate the size of the spine in arbitrary units (see Materials and Methods). Note that the dynamic range of spine sizes exceeds an order of magnitude. $\boldsymbol{B}$, Histogram of all imaged spine sizes of a single neuron on a single day (same as in $\boldsymbol{A} ; n=126)$. C, Same data as in $\boldsymbol{B}$ plotted on a logarithmic scale. Red line shows normal distribution function fitted to the histogram (mean = 1.80; variance $=0.0877$ ). $\boldsymbol{D}$, Log-normal distribution of sizes of all spines. Distribution of the logarithm of the size of the complete dataset of spines across imaging sessions and neurons $(n=3474)$. Red line is a normal distribution function fitted to the histogram (mean $=1.74$; variance $=0.1002$ ).

\section{Results}

To chronically image spines at multiple time points over weeks, we implanted a small glass window in the skull above the auditory cortex of adult transgenic mice of the GFP-M line (Feng et al., 2000). These mice express GFP in a sparse subset of cortical neurons, primarily in layer 5 . We used two-photon laser scanning microscopy to image the same apical dendrites for six time points at intervals of $4 \mathrm{~d}$ (see Materials and Methods). The apical den- dritic trees of the imaged neurons were reconstructed from low magnification in vivo images and were likely to belong to layer 5 neurons given the expression pattern of the GFP-M line and the fact that the corresponding somata were located $>500 \mu \mathrm{m}$ below the cortical surface (Fig. $1 A)$. To quantify possible changes in the dendritic tree of the neuron over time, we measured the positions of the dendritic end points in relation to invariant landmarks more proximal on the dendrite. We found that the dendritic arborization remained largely stable throughout the imaging period and dendritic tips showed only a slight trend to retract (Fig. $1 B$ ). These results are consistent with previous observations (Trachtenberg et al., 2002). Interested in the dynamics of synapses, we imaged and analyzed the intensity of spines from eight neurons in four mice. For the purpose of this study, we focused our attention on the intensities of 579 "stable" spines from eight neurons in four mice that were observed throughout the experiment. Examples of these spines are marked by arrows in Figure $1 C$, which depicts the same dendrite in the first imaging (top) and the last imaging day (bottom).

\section{Sizes of spines are log-normally distributed}

We observed substantial heterogeneity in the sizes of spines. For example, when considering the distribution of the spine sizes of a single neuron on a single day (Fig. 2A,B), the range of spine sizes spans more than one order of magnitude. Interestingly, the distribution of spine sizes is positively skewed, reminiscent of a lognormal distribution. To better quantify this, we plotted a histogram of the logarithm of spine sizes (Fig. 2C), demonstrating that the size distribution can be well approximated by a log-normal function (Fig. 2C, red line). A similar log-normal distribution was obtained when considering the sizes of all spines from all imaging sessions and all neurons (Fig. 2D).

\section{Small variance in mean spine sizes between neurons}

How similar are the distributions of spine sizes of different neurons? The similarity between the distribution of spines on one neuron on one day (Fig. 2C) and the distribution of all spines on all neurons on all days (Fig. $2 D$ ) suggests that the distributions are similar. Indeed, as shown in Figure $3 A$ (different colors), the distributions of the logarithm of spine sizes of the eight neurons are similar. However, a more careful analysis of these distributions reveals small differences between the neurons. This is illustrated in Figure $3 B$, in which the averages of the logarithm of spine sizes for the dif- 

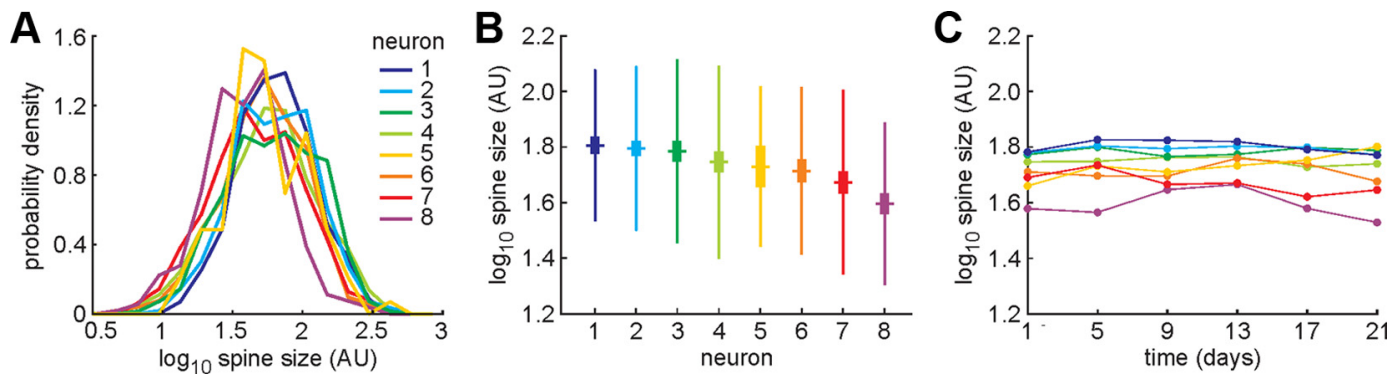

Figure 3. Interneuron and intraneuron variability in spine sizes. $\boldsymbol{A}$, Size distribution of spines across all imaging sessions for eight individual neurons. $\boldsymbol{B}$, Average $\log _{10}$ spine sizes for the eight neurons (thin horizontal lines), SEM (thick vertical lines), and SD (thin vertical lines). Mean logarithm spine sizes show small but significant variations. Note that interneuron variability is significantly larger than intraneuron variability. $\boldsymbol{C}$, Mean of the logarithm of the sizes of spines does not change significantly over days. Numbers of observations for $\boldsymbol{A}-\boldsymbol{C}$ (number of spines $\times 6$ images per spine) are $n=480,756,468,546,96,348,420$, and 360 for neurons $1-8$, respectively.

ferent neurons are denoted by thin horizontal lines and the SEMs are denoted by thick vertical lines. We found that the average size of spines varies significantly between neurons (one-way ANOVA $p<$ $0.0002)$. However, the intraneuron variability in spine size as quantified using the SD (Fig. 3B, thin vertical lines) is much larger than the interneuron variability. More precisely, our analysis reveals that only $5 \%$ of the variability in the logarithm of spine sizes of the full dataset of spines can be attributed to the interneuron variability (interneuron variance, 0.0049; variance of full dataset, 0.10 ).

To what extent does the distribution of spine sizes of individual neurons change over time? To quantify this, we calculated the mean logarithm of spine size for each neuron over time (Fig. $3 C$ ). There was no significant change in the average logarithm of spine size in any of the neurons (all $p$ values for all eight neurons were larger than 0.08 , one-way ANOVA).

\section{Temporal dynamics of spine sizes}

What are the synaptic dynamics that lead to the log-normal distribution of spine sizes? To address this question, we studied how spine sizes change over time. Several characteristic examples are shown in Figure $4 A$. The sizes of these spines over time are depicted in Figure $4 B$. We found that spines undergo substantial changes in their sizes: over the course of $20 \mathrm{~d},>70 \%$ of the spines changed their size by at least $50 \%$, suggesting that synaptic dynamics in the adult cortex are a very active process.

As depicted in Figure 4, $A$ and $B$, the larger a spine is, the larger the change in its size in consecutive imaging days is. To quantify this relation between spine size and its volatility, we conducted the following analysis: we considered all individual size measurements of the spines in the first five imaging sessions ( 579 spines $\times$ 5 images per spine $=2895$ observations). We binned these spine measurements into 200 groups, each containing 14 or 15 measurements of approximately equal spine size. For each observation, we computed the absolute change in spine size to the next imaging session and averaged this number for each group. The results of this analysis are depicted in Figure $4 C$, where we plot the

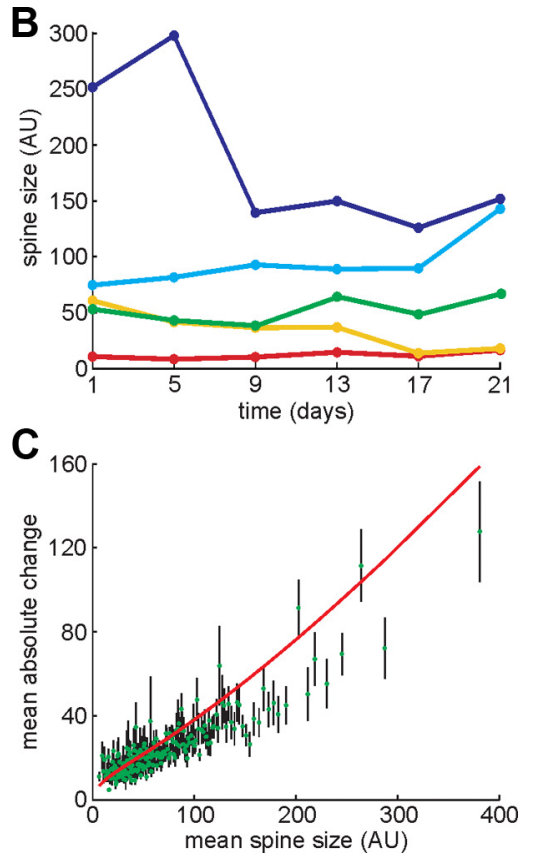

Figure 4. Changes in spine sizes over time are multiplicative. $\boldsymbol{A}$, Examples of spines from neuron 4 , imaged at six time points at A interval. $\boldsymbol{B}$, Sizes of these spines over the six imaging sessions. The color of the line corresponds to the color of the frame spine images shown in $A$. C Mean absolute change in spine size as a function of its size Spine size measurements of imaging sessions $1-5(n=2895)$ were grouped into 200 bins according to their size. For each group, the mean absolute change in spine size to the next imaging session is shown as a function of mean spine size for that group (green dots). Black error bars represent SEM. Red line, Predicted dependence of absolute change on spine size; see Equation 5 in Materials and Methods.

average absolute change in spine size as a function of average spine size for each group. We found that changes in spine size are approximately proportional to the size of the spines. In other words, changes in spine sizes are multiplicative.

Is the finding of multiplicative dynamics linked to the stationary log-normal distribution of spine sizes? It is generally known that a log-normal distribution naturally emerges from multiplicative dynamics. To understand why, we denote by $W$ and $W^{\prime}$ the sizes of a spine in two consecutive imaging sessions. We consider a dynamic process in which changes in spine size are multiplicative and stochastic: $\Delta W=W^{\prime}-W=W \cdot z$, where $z$ is a stochastic variable. In other words, $W^{\prime}=W \cdot(1+z)$. Hence, $\log \left(W^{\prime}\right)=$ $\log (W)+\log (1+z)$. Iterating this process $t$ times, $\log (W(t))=$ $\log (W(0))+\sum_{j=1}^{t} \log (1+z(j))$, where $W(t)$ denotes the size of the spine after $t$ iterations, $W(0)$ denotes its original size, and $z(j)$ denotes the value of the stochastic variable $z$ on the $j$ th iteration. By the Central Limit Theorem, the sum of independent random 


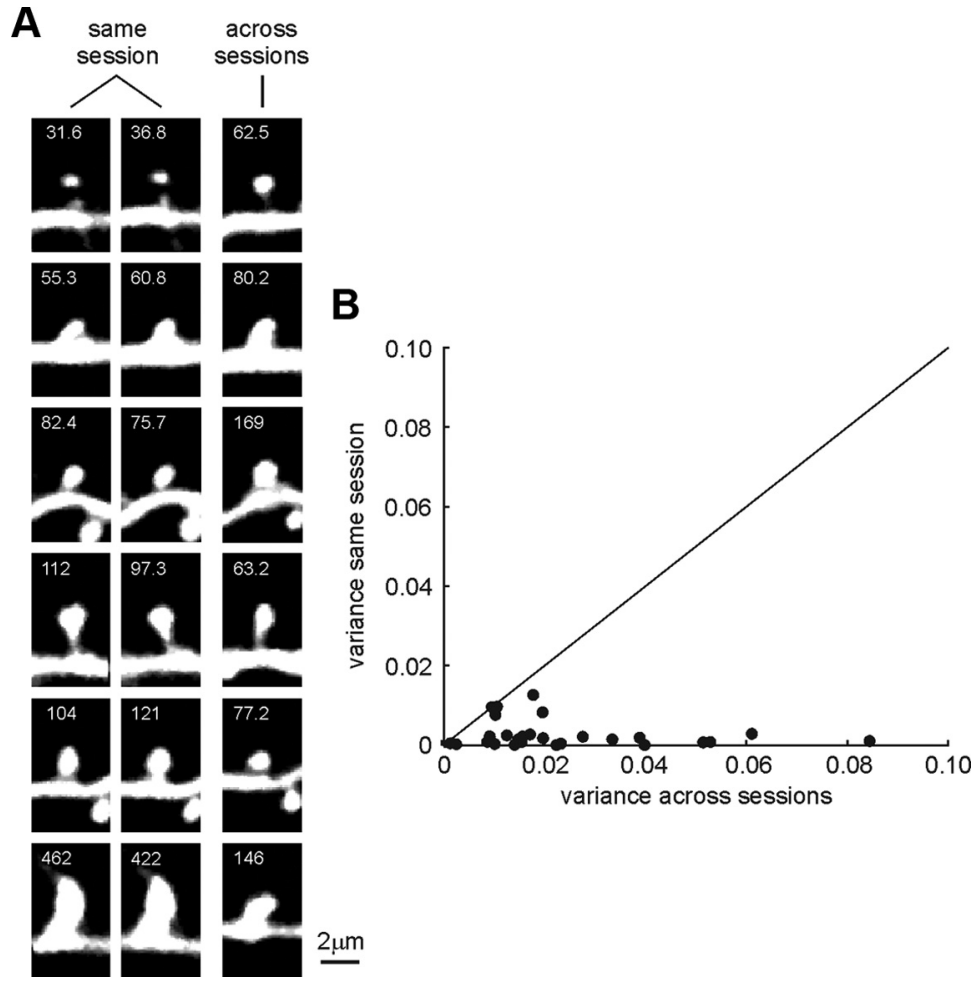

Figure 5. Estimation of the measurement noise. $A$, Examples of spines imaged twice during the same imaging session $(\sim 2-3$ min interval, due to overlapping tiling of image stacks along a given dendrite) and again in a different imaging session ( $\geq 4 \mathrm{~d}$ ). Estimated spine size in arbitrary units is indicated above spines. The variability within one session is most likely due to measurement noise. In most cases, the changes across imaging sessions are much larger than those within a single session. $\boldsymbol{B}$, Plot of variance of spine size across sessions against the variance observed within a session for 27 spines. Black line indicates line of identity. Again, the variance across sessions is typically much larger than the variance within one session.

variables from the same distribution converges to a normal distribution. Therefore, if $t$ is large enough and the stochastic variables $z$ are independent and are drawn from the same distribution, the distribution of $\log (W(t))$ will be normal (see also Song et al., 2005).

\section{A model of spine size dynamics}

The observation that changes in spine sizes are multiplicative (Fig. 4C) provides insights into the emergence of the log-normal distribution of spine sizes in the neocortex (Figs. 2, 3). However, the simple dynamics described above are inconsistent with a stationary size distribution because they predict that the variance of the log-normal distribution will increase with the number of stochastic variables added to the spine size. In contrast, our data indicate that the variance of the spine size distribution is constant over time (data not shown). Therefore, we sought a more accurate description of the temporal dynamics of spines.

As a preliminary step toward a quantitative model of spine size dynamics, we estimated the contribution of measurement noise to the variability and changes in the observed spine sizes. We computed the variability in the logarithm of the measured size of spines measured on the same day, at an interval of a few minutes, and compared it to the between-session variability. Six characteristic examples are presented in Figure 5A, indicating that between-session variability is significantly larger than the withinsession variability, which is most likely due to measurement noise. To further quantify the contribution of the measurement noise to the session to session changes in the measured size of spines, we compared the within-session variability to the between-session variability of the 27 stable spines (Fig. 5B) that were imaged twice in the same session (see Materials and Methods). These results further indicate that the measurement noise is substantially lower than the between-session variability. The within-session measurements indicate that the variance of the measurement noise is $\sigma_{n}^{2}=2.74 \cdot 10^{-3}$, contributing $<3 \%$ to the observed variance in the logarithm of spine sizes (see Materials and Methods). This estimation of measurement noise captures major sources of movement in the brain due to breathing and heart pulsation. However, there could be additional sources of measurement noise that are not accounted by the repeated measurement depicted in Figure 5, e.g., slight differences in the imaging angle between sessions. In the Materials and Methods section, we discuss the consequences of a possible underestimation of measurement noise.

Next, we computed the temporal covariance of the logarithm of spine sizes over the six imaging sessions (Fig. 6A; see Materials and Methods). We found that the covariance decays steeply within $4 \mathrm{~d}$ (one imaging session), followed by a shallower decay. This difference in the decay rates suggests that the dynamics contain more than a single timescale. We sought a phenomenological model of spine dynamics that captures the main observation described in the previous sections, namely (1) the stationary distribution of spine sizes is log-normal (Figs. 2, 3), (2) changes in spine sizes are multiplicative (Fig. 4C), and (3) their temporal correlations are characterized by at least two timescales (Fig. $6 \mathrm{~A}$ ). We posit that the dynamics of the logarithm of the size of a spine are a sum of two independent Ornstein-Uhlenbeck processes (Eq. 1 in Materials and Methods). Moreover, we assume that the logarithm of the observed spine size is equal to the sum of the logarithm of the spine size and the measurement noise, as estimated above (Fig. 5; see also Materials and Methods).

An Ornstein-Uhlenbeck process is a linear stochastic equation that is commonly used to describe stationary Markov processes (Van Kampen, 2007). It is similar to a random walk process in which random events accumulate with time. However, in contrast to the random walk process, the Ornstein-Uhlenbeck process describes continuous dynamics in which the contributions of past steps to the current state decay exponentially. It is characterized by the degree of volatility, $\sigma$, and a single timescale, $\tau$, the time constant of the exponent.

The parameters of the model were obtained by fitting the stationary distribution of Equation 1 to the covariance plotted in Figure $6 \mathrm{~A}$ and the stationary distribution of spine sizes (Fig. 2D) (see Materials and Methods). The resultant two timescales are as follows: $\tau_{1}=$ $212 \mathrm{~d}$ and $\tau_{2}=2.87 \mathrm{~d}$; and the magnitudes of the volatility terms are as follows: $\sigma_{1}^{2}=0.0683$ and $\sigma_{2}^{2}=0.0292$.

How well does this simple model capture our main observations described above? (1) The stationary distribution of an Ornstein-Uhlenbeck process is normal (Fig. 2 D, red line). Therefore, the model can account for the normal distribution of the loga- 

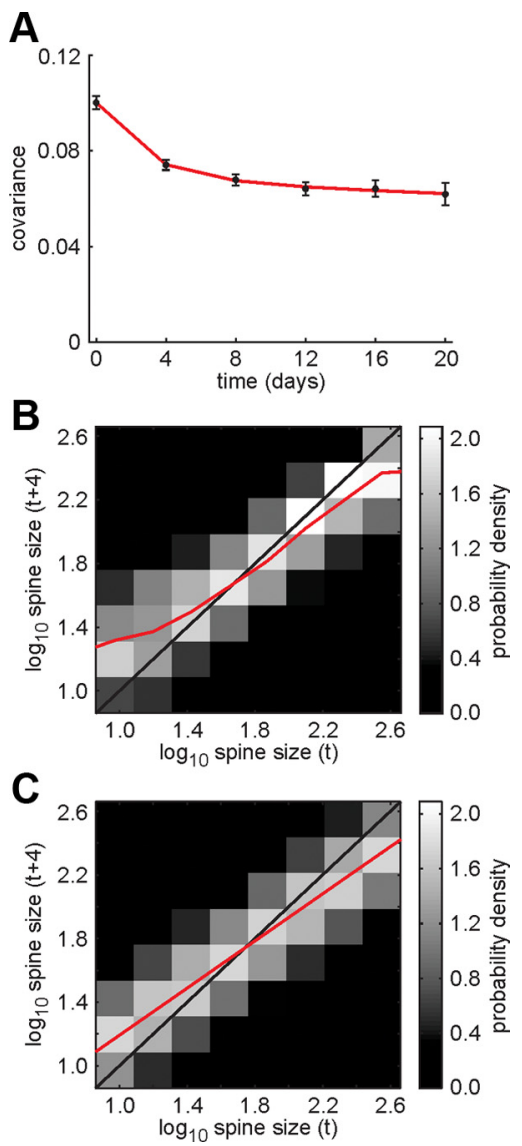

Figure 6. Quantitative analysis of the temporal dynamics of "stable" spine sizes. A, Black dots, covariance of spine sizes across imaging sessions. Error bars are SEM. Red line indicates covariance fitted by our model (Eq. 2 in Materials and Methods). $\boldsymbol{B}$, Empirically measured transition matrix: abscissa is the size of the spine in one imaging session, ordinate is the size in the following imaging session, and color corresponds to the probability density; black line is line of identity. Red line shows average expectation value of size in the next imaging session. C, Same as in $\boldsymbol{B}$ based on our model, Equation 3 in Materials and Methods.

rithm of spine sizes. (2) The temporal dynamics of the model (Fig. $4 C$, red line) are consistent with the multiplicative dynamics (Fig. 4C, dots). (3) The model fits well the empirically observed temporal correlations (Fig. 6A, red line).

Moreover, the model provides us with a detailed probabilistic description of the dynamics of spine sizes. In particular, assuming that the two Ornstein-Uhlenbeck processes have reached equilibrium, we can use the model to predict how a spine is expected to change over time, given its initial size. To test how well our model captured these transitions, we computed the empirical transition matrix of spine sizes of our dataset (Fig. $6 \mathrm{~B}$ ). The abscissa corresponds to the logarithm of the size of the spine at time $t$ and the ordinate denotes the logarithm of the size of the spine on the next imaging session, $t+4 \mathrm{~d}$. The gray values code for the probability density. Note that nonzero probability densities are centered along the identity line (black line), indicating that changes in spine sizes are small compared with the dynamic range of spine sizes. The red line indicates the expectation value of the logarithm of the spine size in the next imaging session as a function of its size in the previous imaging session. Note that the red line is above the identity line for small spines and below it for large spines. This means that large spines tend to become smaller, whereas small ones tend to increase. In other words, on average, spines tend to contract to a medium size, the intersection of the line of identity with the average line. Interestingly, these results, namely volatility of spines/synaptic efficacies and their tendency to contract to a medium size, were previously observed in neuronal cultures and were shown there to depend on network activity (Yasumatsu et al., 2008; Minerbi et al., 2009).

Similarly, we computed the predicted transition matrix based on our model (Fig. 6C). We found that, similar to the empirical transition matrix, the highest probabilities were centered near the line of identity. Moreover, our model captures the tendency of spines to contract, as is evident from the slope of the red line. In summary, our results show that the multiplicative spine dynamics that give rise to the stationary log-normal distribution can be captured remarkably well using a simple model based on two independent Ornstein-Uhlenbeck processes.

\section{Discussion}

In this paper, we used chronic imaging of spines in the auditory cortex to study the dynamics of neuronal connections in the living brain. Our main findings are as follows: (1) the distribution of spine sizes is log-normal; (2) changes in spine size over time are multiplicative, which explains the emergence of the log-normal distribution; and (3) the dynamics of the sizes of spines are characterized by two timescales and are captured by a sum of two Ornstein-Uhlenbeck processes.

\section{Log-normal distribution of spine sizes}

Whereas most previous studies have focused on a binary description of spine dynamics (i.e., presence/absence of a spine), we were interested in the distribution and dynamics of spine sizes, since they can serve as a proxy for the strength of synaptic connections (Harris and Stevens, 1989; Matsuzaki et al., 2001, 2004; Holtmaat et al., 2005; Knott et al., 2006; Kopec et al., 2006; Arellano et al., 2007). We found that the spine sizes of a single neuron can vary over almost two orders of magnitude (Fig. 2), implying that a binary description of a spine as present or absent is incomplete.

Interestingly, we found that spine sizes are distributed in a log-normal manner and this distribution remains stable over time. This observation supports the hypothesis that synaptic efficacies in the cortex are log-normally distributed. This hypothesis is also supported by several previous studies using different experimental techniques. Cortical spines of layer $2 / 3$ pyramidal neurons of a mouse visual cortex were analyzed using a combination of gold-toned Golgi impregnations and serial thin-section electron microscopy (Arellano et al., 2007). The distribution of spine volumes, averaged over several pyramidal neurons, was found to be broad and positively skewed. Although the exact shape of the distribution was not further examined and the number of spines analyzed was limited, qualitatively, their distribution resembles our Figure $2 B$. In addition, paired recordings in acute brain slices of rat visual cortex (Song et al., 2005) and barrel cortex (Feldmeyer et al., 2002) demonstrated that the distribution of connection strengths between neurons is positively skewed. These distributions were approximated using a lognormal function (Song et al., 2005; Sarid et al., 2007). Note, however, that the connection strength between a pair of neurons as estimated by evoked EPSP reflects the contribution of a variable number of synapses, typically 4-8 (Markram et al., 1997), whereas spines in the cortex typically correspond to single synapses (Harris and Stevens, 1989; Knott et al., 2006). Interestingly, the amplitude distribution of miniature EPSCs in cultured neurons, which are believed to originate from single synapses, is also positively skewed (Bekkers and Stevens, 1995; van Rossum et al., 2000). 
We would like to note that the distribution of motor unit sizes is also reminiscent of a log-normal function (Betz et al., 1979; Taxt, 1983a,b; Lu et al., 2009), which raises the possibility that a log-normal distribution of synaptic strength observed in cortical neurons represents a more general principle of organization in the nervous system.

\section{Similar distribution of spine sizes across neurons}

Assuming that the size of a spine is correlated with synaptic efficacy, this study provides detailed quantitative information about the distribution of synaptic efficacies onto single neurons and the variability of the distributions across neurons. In this section, we use this information to challenge a recent theory that relates the distributions of synaptic efficacies and neuronal activities in the cortex.

The distribution of spontaneous firing rates in the primary auditory cortex is positively skewed and has been approximated by a log-normal function (Hromádka et al., 2008). A recent intriguing theoretical study by Koulakov et al. (2009) suggested that the log-normal distributions of connections and firing rates are closely related. The basic idea of the model is that log-normally distributed firing rates, when combined with a Hebbian plasticity rule, are expected to result in a log-normal distribution of (1) all synaptic weights in the network, (2) all synaptic weights onto single neurons, and (3) the mean synaptic weights onto individual neurons. This model is self-consistent, because such a distribution of mean synaptic efficacies onto individual neurons, in turn, would result in a log-normal distribution of firing rates. Importantly, the model predicts that the variance of the distribution of the logarithm of the firing rates and the variance of the distribution of the logarithm of the synaptic connections onto individual neurons are comparable. By measuring the sizes of many spines onto individual neurons, we can test these predictions. (1) The log-normal distribution of all synaptic weights in the network is consistent with our data (Fig. 2D). (2) Our finding that the log-normal distribution of synaptic weights applies also for single neurons (Figs. 2C, $3 A$ ) is also consistent with the model. (3) We also observed statistically significant differences in the mean synaptic sizes of spines across neurons. However, the differences in spine sizes between our imaged neurons are not consistent with the model: the variance of the distribution of the mean logarithm $\left(\log _{10}\right)$ of spine sizes of individual neurons in our dataset is only 0.005 . This is more than an order of magnitude smaller than the variance in the firing rates, 0.2 , as estimated from Figure 3C in Hromádka et al. (2008). This discrepancy is a serious challenge to their model. One possible explanation could be that our data are restricted to layer 5 pyramidal neurons, whereas the measured spontaneous firing rates were probably obtained from several cell types in various layers.

One approach to reconcile the approximate homogeneity of mean synaptic efficacies and the large heterogeneity in spontaneous firing rates is based on the theory of balanced networks. According to this theory, excitation and inhibition are balanced and the firing of individual spikes results from fluctuations in the synaptic input. As a result, even minute heterogeneities in the total number of synapses would lead to a broad distribution of firing rates (van Vreeswijk and Sompolinsky, 1996). Thus, the observed small imbalances in the mean synaptic inputs could result in a long-tail distribution of the firing rates. An alternative possibility is that the broad distribution of firing rates results from heterogeneities in the integrating or biophysical properties of neurons (see also van Vreeswijk and Sompolinsky, 1998).

\section{The emergence of log-normal distribution from multiplicative dynamics}

In most models of synaptic plasticity, potentiation and depression change the synaptic weight by adding or subtracting a fixed amount that is independent of the synaptic weight (Hopfield, 1982; Blum and Abbott, 1996; Gerstner et al., 1996; Kempter et al., 1999; Song et al., 2000). In other words, changes in synaptic efficacies are additive. Additive changes at timescales of minutes are supported by several in vitro studies (Kopec et al., 2006). Often, a decay term is also incorporated (Kistler and van Hemmen, 2000; Gütig et al., 2003) to prevent the synapse from reaching saturation values (Dayan and Abbott, 2001). The stationary distribution of synaptic weights in these models typically falls into one of two categories: (1) Competition between synapses, if sufficiently strong, leads to a bimodal distribution of synaptic efficacies. Such competition can result from Hebbian plasticity. (2) In contrast, in the absence of such competition, these models typically yield a normal distribution of synaptic efficacies. Thus, these studies imply that to explain the log-normal distribution of spine sizes, we should revise these synaptic plasticity rules and consider the possibility that synaptic changes are directly dependent on the synaptic efficacy, beyond the decay term (van Rossum et al., 2000; Morrison et al., 2007; Koulakov et al., 2009). An interesting study demonstrated multiplicative scaling of synaptic efficacies as a result of homeostatic plasticity (Turrigiano et al., 1998). However, this homeostatic scaling is a global mechanism that affects all synapses in a similar fashion, whereas in our dataset individual synapses of the same neuron changed in opposite directions.

The emerging picture from this study is that the network architecture is highly volatile, even in primary sensory regions such as the auditory cortex. However, there is a structure to this dynamics: changes are multiplicative, which appears to be a major determinant of the distribution of synaptic efficacies in the cortex.

\section{References}

Arellano JI, Benavides-Piccione R, Defelipe J, Yuste R (2007) Ultrastructure of dendritic spines: correlation between synaptic and spine morphologies. Front Neurosci 1:131-143.

Bekkers JM, Stevens CF (1995) Quantal analysis of EPSCs recorded from small numbers of synapses in hippocampal cultures. J Neurophysiol 73:1145-1156.

Betz WJ, Caldwell JH, Ribchester RR (1979) The size of motor units during post-natal development of rat lumbrical muscle. J Physiol 297:463-478.

Bhatt DH, Zhang S, Gan WB (2009) Dendritic spine dynamics. Annu Rev Physiol 71:261-282.

Blum KI, Abbott LF (1996) A model of spatial map formation in the hippocampus of the rat. Neural Comput 8:85-93.

Dayan P, Abbott LF (2001) Theoretical neuroscience: computational and mathematical modeling of neural systems. Cambridge, MA: MIT.

Feldmeyer D, Lübke J, Silver RA, Sakmann B (2002) Synaptic connections between layer 4 spiny neurone-layer $2 / 3$ pyramidal cell pairs in juvenile rat barrel cortex: physiology and anatomy of interlaminar signalling within a cortical column. J Physiol 538:803-822.

Feng G, Mellor RH, Bernstein M, Keller-Peck C, Nguyen QT, Wallace M, Nerbonne JM, Lichtman JW, Sanes JR (2000) Imaging neuronal subsets in transgenic mice expressing multiple spectral variants of GFP. Neuron 28:41-51.

Gerstner W, Kempter R, van Hemmen JL, Wagner H (1996) A neuronal learning rule for sub-millisecond temporal coding. Nature 383:76-81.

Grutzendler J, Kasthuri N, Gan WB (2002) Long-term dendritic spine stability in the adult cortex. Nature 420:812-816.

Gütig R, Aharonov R, Rotter S, Sompolinsky H (2003) Learning input correlations through nonlinear temporally asymmetric Hebbian plasticity. J Neurosci 23:3697-3714

Harris KM, Stevens JK (1989) Dendritic spines of CA 1 pyramidal cells in 
the rat hippocampus: serial electron microscopy with reference to their biophysical characteristics. J Neurosci 9:2982-2997.

Hofer SB, Mrsic-Flogel TD, Bonhoeffer T, Hübener M (2009) Experience leaves a lasting structural trace in cortical circuits. Nature 457:313-317.

Holtmaat A, Svoboda K (2009) Experience-dependent structural synaptic plasticity in the mammalian brain. Nat Rev Neurosci 10:647-658

Holtmaat AJ, Trachtenberg JT, Wilbrecht L, Shepherd GM, Zhang X, Knott GW, Svoboda K (2005) Transient and persistent dendritic spines in the neocortex in vivo. Neuron 45:279-291.

Holtmaat A, Wilbrecht L, Knott GW, Welker E, Svoboda K (2006) Experience-dependent and cell-type-specific spine growth in the neocortex. Nature 441:979-983.

Holtmaat A, Bonhoeffer T, Chow DK, Chuckowree J, De Paola V, Hofer SB, Hübener M, Keck T, Knott G, Lee WC, Mostany R, Mrsic-Flogel TD, Nedivi E, Portera-Cailliau C, Svoboda K, Trachtenberg JT, Wilbrecht L (2009) Long-term, high-resolution imaging in the mouse neocortex through a chronic cranial window. Nat Protoc 4:1128-1144.

Hopfield JJ (1982) Neural networks and physical systems with emergent collective computational abilities. Proc Natl Acad Sci U S A 79: $2554-2558$.

Hromádka T, Deweese MR, Zador AM (2008) Sparse representation of sounds in the unanesthetized auditory cortex. PLoS Biol 6:e16.

Katz Y, Menon V, Nicholson DA, Geinisman Y, Kath WL, Spruston N (2009) Synapse distribution suggests a two-stage model of dendritic integration in CA1 pyramidal neurons. Neuron 63:171-177.

Kempter R, Gerstner W, van Hemmen JL (1999) Hebbian learning and spiking neurons. Phys Rev E Stat Phys Plasmas Fluids Relat Interdiscip Topics 59:4498-4514.

Kistler WM, van Hemmen JL (2000) Modeling synaptic plasticity in conjuction with the timing of pre- and postsynaptic action potentials. Neural Comput 12:385-405.

Knott GW, Holtmaat A, Wilbrecht L, Welker E, Svoboda K (2006) Spine growth precedes synapse formation in the adult neocortex in vivo. Nat Neurosci 9:1117-1124.

Kopec CD, Li B, Wei W, Boehm J, Malinow R (2006) Glutamate receptor exocytosis and spine enlargement during chemically induced long-term potentiation. J Neurosci 26:2000-2009.

Koulakov AA, Hromádka T, Zador AM (2009) Correlated connectivity and the distribution of firing rates in the neocortex. J Neurosci 29:3685-3694.

Lu J, Tapia JC, White OL, Lichtman JW (2009) The interscutularis muscle connectome. PLoS Biol 7:e32.

Majewska AK, Newton JR, Sur M (2006) Remodeling of synaptic structure in sensory cortical areas in vivo. J Neurosci 26:3021-3029.

Markram H, Lübke J, Frotscher M, Roth A, Sakmann B (1997) Physiology and anatomy of synaptic connections between thick tufted pyramidal neurones in the developing rat neocortex. J Physiol 500:409-440.

Matsuzaki M, Ellis-Davies GC, Nemoto T, Miyashita Y, Iino M, Kasai H (2001) Dendritic spine geometry is critical for AMPA receptor expression in hippocampal CA1 pyramidal neurons. Nat Neurosci 4: $1086-1092$.
Matsuzaki M, Honkura N, Ellis-Davies GC, Kasai H (2004) Structural basis of long-term potentiation in single dendritic spines. Nature 429:761-766.

Minerbi A, Kahana R, Goldfeld L, Kaufman M, Marom S, Ziv NE (2009) Long-term relationships between synaptic tenacity, synaptic remodeling, and network activity. PLoS Biol 7:e1000136.

Morrison A, Aertsen A, Diesmann M (2007) Spike-timing-dependent plasticity in balanced random networks. Neural Comput 19:1437-1467.

Oray S, Majewska A, Sur M (2004) Dendritic spine dynamics are regulated by monocular deprivation and extracellular matrix degradation. Neuron 44:1021-1030.

Sarid L, Bruno R, Sakmann B, Segev I, Feldmeyer D (2007) Modeling a layer 4 -to-layer $2 / 3$ module of a single column in rat neocortex: interweaving in vitro and in vivo experimental observations. Proc Natl Acad Sci U S A 104:16353-16358.

Song S, Miller KD, Abbott LF (2000) Competitive Hebbian learning through spike-timing-dependent synaptic plasticity. Nat Neurosci 3:919-926.

Song S, Sjöström PJ, Reigl M, Nelson S, Chklovskii DB (2005) Highly nonrandom features of synaptic connectivity in local cortical circuits. PLoS Biol 3:e68.

Taxt T (1983a) Motor unit numbers, motor unit sizes and innervation of single muscle fibres in hyperinnervated adult mouse soleus muscle. Acta Physiol Scand 117:571-580.

Taxt T (1983b) Cross-innervation of fast and slow-twitch muscles by motor axons of the sural nerve in the mouse. Acta Physiol Scand 117:331-341.

Trachtenberg JT, Chen BE, Knott GW, Feng G, Sanes JR, Welker E, Svoboda $\mathrm{K}$ (2002) Long-term in vivo imaging of experience-dependent synaptic plasticity in adult cortex. Nature 420:788-794.

Turrigiano GG, Leslie KR, Desai NS, Rutherford LC, Nelson SB (1998) Activity-dependent scaling of quantal amplitude in neocortical neurons. Nature 391:892-896.

Van Kampen NG (2007) Stochastic processes in physics and chemistry. Amsterdam: Elsevier.

van Rossum MC, Bi GQ, Turrigiano GG (2000) Stable Hebbian learning from spike timing-dependent plasticity. J Neurosci 20:8812-8821.

van Vreeswijk C, Sompolinsky H (1996) Chaos in neuronal networks with balanced excitatory and inhibitory activity. Science 274:1724-1726.

van Vreeswijk C, Sompolinsky H (1998) Chaotic balanced state in a model of cortical circuits. Neural Comput 10:1321-1371.

Xu T, Yu X, Perlik AJ, Tobin WF, Zweig JA, Tennant K, Jones T, Zuo Y (2009) Rapid formation and selective stabilization of synapses for enduring motor memories. Nature 462:915-919.

Yang G, Pan F, Gan WB (2009) Stably maintained dendritic spines are associated with lifelong memories. Nature 462:920-924.

Yasumatsu N, Matsuzaki M, Miyazaki T, Noguchi J, Kasai H (2008) Principles of long-term dynamics of dendritic spines. J Neurosci 28:13592-13608.

Zuo Y, Lin A, Chang P, Gan WB (2005) Development of long-term dendritic spine stability in diverse regions of cerebral cortex. Neuron 46:181189. 